\title{
Nutzerzentrierte Entwicklung einer ortsunabhängigen Maschinenabnahme mittels Augmented Reality
}

Nedim Kovacevic, Jantje Meinzer, Rainer Stark

Die Nutzung von Augmented Reality (AR) stellt eine Vielzahl von Lösungsansätzen für Herausforderungen unterschiedlicher Bereiche, z.B. der Industrie, Medizin, Unterhaltungsmedien und Bildung, zur Verfügung. Speziell werden hier die Möglichkeiten von AR in der Fertigungsindustrie, genauer bei der Abnahme elektrischer Antriebe der Siemens AG, betrachtet. Dabei werden unter anderem messbare Größen wie Drehmoment, Ankerstrom, -spannung, Drehzahl, Schwingungen, Fertigungstoleranzen etc. gemessen, aber auch nicht quantifizierbare Größen wie Sichtprüfungen von Schweißnähten und Lackierung in Betracht gezogen. Angestrebt wird eine Methode, welche es zukünftig ermöglicht, die Inbetriebnahme ortsunabhängig zu verfolgen und Inspektionen aus der Ferne durchzuführen. Die Herausforderung liegt dabei darin, eine zumindest gleichwertige, wenn nicht sogar verbesserte Erfahrung und Prüfergebnisvalidität für die Beteiligten gegenüber der vor Ort Abnahme zu erzeugen. Im Rahmen des Forschungsvorhabens wurde in interdisziplinärer Zusammenarbeit zwischen Ingenieur/-innen und Sozialwissenschaftler/-innen ein Demonstrator-Konzept für eine ortsunabhängige Maschinenabnahme entwickelt.

Keywords: Augmented Reality (AR), Maschinenabnahme, Nutzerzentrierung; User-CentredDesign, Digitaler Zwilling, Industrie 4.0

\section{Einleitung}

Die Nutzung (teil-) virtueller Räume eröffnet Industrie und Arbeitsalltag neue Gestaltungsmöglichkeiten. AR-Technologie ergänzt eine vorhandene Realität durch Elemente einer virtuellen Realität in Echtzeit (Mehler-Bicher \& Steige, 2014). So vermag AR-Technologie Wertschöpfungsketten ganzheitlich zu begleiten und verschiedene Anschlussmöglichkeiten in der Fertigung zu finden. Das Forschungsvorhaben Elektrische Antriebe 2.0 (EA 2.0) betrachtet beispielsweise sowohl Produkt- als auch Prozessinnovationen entlang der Wertschöpfungskette einer elektrischen Maschine sowie die Technologien neue Werkstoffe, additive Fertigung und Digitalisierung (WvSC, 2020). Ein Teilprojekt erarbeitet einen Service zur ortsunabhängigen Maschinenabnahme durch die Verwendung von AR-Technologien. Durch neue Technologien stehen Prüfdaten aus einem digitalen Zwilling zur Verfügung und ersetzen die Arbeit mit Dokumenten in Papierform. 
Um den einwandfreien Betrieb von elektrischen Antrieben zu gewährleisten, werden diese nach der Fertigung in einem Prüffeld durch Kund/-innen und/oder eine von innen beauftragte Prüfgesellschaft auf unterschiedliche Kriterien vor Ort getestet. Zu den Kriterien zählen messbare Größen wie Drehmoment, Ankerstrom, -spannung, Drehzahl, Schwingungen oder Fertigungstoleranzen. Zusätzlich werden nicht quantifizierbare Größen betrachtet wie Schweißnähte und Lackierung durch Sichtprüfungen. Neben der Qualitätssicherung, dem Nachweis der Auftragserfüllung und Funktionstüchtigkeit der Maschine vor der finalen Inbetriebnahme, erfüllt der Prüfprozess laut des Anwenderunternehmens Siemens wichtige Aspekte der Kundenbindung und des Kundenerlebnisses. Mit Hilfe von AR-Technologien soll die technische Prüfung durch Visualisierung zusätzlicher Wissensressourcen und geeigneter Daten übersichtlicher informativer und ortsunabhängig gestaltet werden. Die Herausforderung einer ortsunabhängigen Maschinenabnahme liegt darin, eine zumindest gleichwertige, wenn nicht sogar verbesserte Erfahrung und Prüfergebnisvalidität für die Beteiligten gegenüber der vor Ort Abnahme zu erzeugen. Nachfolgend wird ein Überblick über relevante Studien zu AR-Technologien gegeben, deren Erkenntnisse als Grundlage für ein Konzept dienen, das die oben genannten Herausforderungen im Projekt EA2.0 adressiert. Da die Abnahme einen bedeutenden Teil des Kundenerlebnisses darstellt, geschieht die Entwicklung nicht ausschließlich aus Perspektive der technischen Machbarkeit. Sozial- und Ingenieurwissenschaft wählten in interdisziplinärer Zusammenarbeit ein nutzerzentriertes Vorgehen, das neben der technischen Machbarkeit auch die Nutzerperspektive in das hier vorgestellte Konzept einbindet.

\section{Stand der Technik}

Eine Vielzahl an Arbeiten, so zeigt es bspw. die Übersicht von Mura \& Dini (2015), beschäftigt sich mit den Potentialen von AR in der Industrie. Das hier vorgeschlagene Konzept einer AR-unterstützten Abnahme lässt sich in den Bereich Inspektion und Wartung einordnen. Die Überblendung von Informationen innerhalb einer Inspektion ermöglicht es Menschen, Arbeitsschritte schneller durchzuführen. So erwirkt der Einsatz von AR-Technologie einen Vorteil. Zusätzlich ermöglicht die Überblendung den Blick durchgängig auf die zu bearbeitende Aufgabe zu richten, ohne die Blickrichtung zwischen analogen Dokumenten und der Aufgabe zu wechseln. Polvi, Taketomi, \& Motek (2018) entwickelten eine AR-App für Mobilgeräte, welche die Inspektion von PCHardware unterstützt. Anhand der App wurde der Vorteil von AR-Assistenzsystemen überprüft. Hierfür wurden Informationen zu Prozessschritten mittels AR über ein Motherboard geblendet. Zwei Testgruppen $(N=24)$ überprüften die Hardware. Die 
erste Gruppe erledigte die Aufgabe mit Hilfe der AR-Applikation. Die zweite Gruppe bekam eine bildgeführte Dokumentation zur Unterstützung. Das Ergebnis zeigt, dass die AR-Testgruppe weniger Zeit brauchte, weniger Fehler machte und weniger Blickabweichungen vom Objekt verzeichnete als die Gruppe ohne AR-Unterstützung (Polvi, Taketomi, Moteki 2018).

Die Idee, AR für die Inspektion zu nutzen, ist am Ende des 19. Jahrhunderts bereits von Webster, Feiner, Macintyre, Massie, Krueger (1996) erforscht worden. Sie entwickelten einen Augmented Reality Head Mounted Device (HMD)-Prototypen, welcher es ermöglichte, Stützstrukturen über Wänden zu visualisieren, um dementsprechende Restaurationsarbeiten zu planen (Webster, Feiner, Macintyre, Massie, Krueger 1996). Um die Inspektion von Schweißpunkten von Fahrzeugkarosserien zu unterstützen, entwickelten Zhou, Lee, Thomas und Mena (2011) ein Spatial Augmented Reality System (SAR), welches die Information nicht mittels eines Handheld Devices oder HMDs überblendet, sondern einen Projektor benutzt, um die Position und Inspektionsart der Schweißpunkte zu visualisieren. Dadurch werden zwei Prozessschritte gegenüber der herkömmlichen Inspektion gespart, da das Prüfpersonal Schweißpunktort und -prüfart nicht mehr durch den Abgleich von Dokumenten sondieren muss (Zhou, Lee, Thomas, Mena, 2011). Zheng, et al. (2019) entwickelten eine AR-Anwendung zur Inspektion und Wartung von Flugzeugkabeln, um die Anzahl der zu lesenden Dokumente zu verringern. Durch ein "convolutional neural network“ (CNN) wurden die Kabelklammern erkannt und der darauf aufgedruckte Text gelesen. Ausgelöst durch die Erkennung wurde eine Wartungsanleitung überblendet. Im Anwendungsfall werden zur Prüfung von elektrischen Antrieben korrespondierende Sensordaten über das reale Objekt geblendet. In Stark, Kucera, Haffner, Draho, Leskovsky (2020) werden "Internet of things" (IOT)-Daten über einen Roboter geblendet, um den Umgebungszustand des Einsatzortes zu verfolgen. In Revetria, Tonelli, Damiani, Demartini (2019) wird die Verformung eines Metallwinkels mittels einer Wheatstone-Brücke gemessen und anschließend über dem Bauteil visualisiert, damit die mechanische Überlastung überwacht werden kann. Im Anwendungsfall werden zur besseren Überwachung von elektrischen Antrieben korrespondierende Sensordaten über das reale Objekt geblendet.

Schröder, Steinmetz, Garcia, Espindola (2016) stellten eine Methode vor, die ein 3DModell einer Ölbohreinrichtung in eine reale Umgebung projiziert und mit Sensordaten ergänzt (Schröder, Steinmetz, Garcia, Espindola, 2016). Diese Methode eignet sich als Grundlage für die Erweiterung des EA 2.0 Vorhabens der Maschinenabnahme auf eine virtuelle Inbetriebnahme. Das Vorhaben der AR-gestützten Abnahme hebt sich 
insofern von den beschriebenen Studien ab, als dass die Methode ortsunabhängig durchgeführt werden soll und neben der technischen Machbarkeit auch Nutzeranforderungen in Betracht gezogen werden.

\section{Nutzerzentrierte Entwicklung in interdisziplinärer Zusammenarbeit}

Ein nutzerzentriertes Vorgehen strebt nach einem klaren Verständnis der Nutzeranforderungen. Als Gelingfaktoren zählen ein iterativer Prozess, die Evaluierung von Teilergebnissen und eine enge Zusammenarbeit zwischen verschiedenen Disziplinen (Vredenburg, Mao, Smith, Carey, 2002). Vor allem bei der Entwicklung von Technologien, die eine ausgeprägte Interaktion zwischen Menschen und Maschine erfordern, sind neben technisch-wissenschaftlichen Perspektiven auch „sozial-, geistes-, natur- und ingenieurwissenschaftliche Perspektiven und Erkenntnisse" gleichermaßen opportun (BMBF, 2018, S. 6). Darum haben sich Mitarbeiter/-innen aus Sozialwissenschaft und Ingenieurswesen im Projekt EA2.0 zusammengeschlossen, um ein erfolgversprechendes Konzept in interdisziplinärer Zusammenarbeit für das Vorhaben zu entwickeln.

\section{Nutzerzentrierte Entwicklung in der Forschung}

Grund für den Einbezug der Nutzerperspektive ist die Akzeptanzsteigerung der Technologie (Gemser \& Perks, 2015) und die damit verbundene Aussicht einer erfolgreichen Technologieentwicklung durch Verringerung des Marktrisikos (Lüthje, 2007). Der berufliche Alltag wird sichtbar digitaler, mit der Besonderheit gegenüber dem privaten Alltag, dass der Digitalisierungskontext am Arbeitsplatz einen nicht-freiwilligen Charakter trägt (Brown, Massey, Montoya-Weiss, Burkman 2002; Renn, 2005). Neue digitale Technologien, die den Arbeitsalltag vereinfachen oder entlasten sollen, funktionieren nur dann zuverlässig und nachhaltig, wenn potenzielle zukünftige Nutzende z.B. Mitarbeitende diese akzeptieren und tatsächlich nutzen. Gut gemeinte Technologien, die in der Wahrnehmung der Mitarbeitenden als nicht hilfreich, als zusätzliche Belastung oder gar als Bedrohung des eigenen Arbeitsplatzes wahrgenommen werden, verfehlen ihr Ziel (BMBF, 2018; Renn, 2005). Nutzerorientierte Technologieentwicklung und Veränderungsprozesse werden zur Grundvoraussetzung der Technologisierung von Arbeitsplätzen (Kriegesmann \& Kerka, 2014). Dabei geht es um eine Einbindung von Nutzenden möglichst früh und begleitend durch den gesamten Innovationsprozess (Williams, Yao, Nurse, 2020). Eine frühe Einbindung wird z.B. durch eine fundierte Anforderungserhebung oder Nutzeranalyse erfüllt, welche die Berücksichtigung der Nutzerbedarfe schon in der Konzeptions- und Planungsphase umfasst (Trübswetter, 
Figueiredo, Prinz, 2020). Eine mehrdimensionale Perspektive gestattet, getroffene Annahmen über mögliche Nutzergruppen zu überprüfen und zu korrigieren. Nicht nur große Unternehmen wie SAP haben die Wichtigkeit nutzerzentrierter Technologieentwicklungen erkannt (Butz \& Krüger, 2017), sondern auch die Forschungs- und Innovationspolitik. In der Hightech-Strategie verstetigt das Bundesministerium für Bildung und Forschung (BMBF) die partizipative Einbindung von relevanten Akteuren in die Entwicklung von Förderprogrammen (BMBF, 2018). Das hier vorgestellte Vorhaben nutzt Erkenntnisse aus dem User-Centred Design (UCD) zur nutzerzentrierten Entwicklung. Die ersten sechs Monate des Vorhabens waren neben der Erarbeitung eines technischen Konzepts vor allem auch für die Bedarfserhebung der Nutzenden reserviert. Norman \& Draper definierten den Begriff UCD schon Mitte der 1980er als menschenzentrierte Umsetzungsstrategie (Norman \& Draper, 1986). Die DIN EN ISO 9241210 definiert den User-Centred-Design (UCD)- Prozess als menschzentrierte Gestaltung gebrauchstauglicher interaktiver Systeme. Im Mittelpunkt des Gestaltungsprozesses stehen die Anforderungen der späteren Nutzenden, sowie Erkenntnisse und Methoden anderer Disziplinen zur Gebrauchstauglichkeit (Deutsches Institut für Normung, 2011). Der technologische Gestaltungsprozess bezieht Nutzende anhand unterschiedlicher Methoden in den gesamten iterativ aufgebauten Entwicklungsprozess mit ein (BMBF, 2018). Dieses Vorgehen verschafft dem Vorhaben Wissen über den Nutzungskontext der Technologie sowie notwendige funktionale Erwartungen an eben diesen (Trübswetter, Figueiredo, Prinz, 2020).

\section{Nutzerzentrierte Entwicklung in der Praxis}

Zur Erhebung der Nutzerbedarfe wurde ein Vorgehen gewählt ähnlich der von Butz und Krüger (2017) beschriebenen Methode. Sie unterteilen den UCD-Prozess - angelehnt an Benyon (2010) - in vier Phasen (Verstehen, Designen, Vergegenwärtigen, Evaluieren), die nicht linear, sondern im Wechselspiel stattfinden (Butz \& Krüger, 2017). Der Designprozess fällt an dieser Stelle weg, da die Arbeit nur die Konzepterarbeitung betrachtet. Zu Beginn sei gesagt, dass persönliche Bedürfnisse und daraus resultierende Anforderungen einen subjektiven Charakter innehaben, dennoch wird über Kategorisierung und Evaluierung der Angaben ein gewisses Level an Objektivität angestrebt (Butz \& Krüger, 2017), das keinen Anspruch auf wissenschaftliche Allgemeingültig erhebt. Durch dieses Vorgehen kann eine auf die spezifischen Bedürfnisse der Akteursgruppen abgestimmte Entwicklung der Technologie erzielt werden.

Verstehen: Zunächst wurden relevante Akteure identifiziert, deren Bedarfe es zu verstehen galt. Hüsing et al. (2002) macht einen Vorschlag zur Akteursliste: Gegebenenfalls die Öffentlichkeit, Verantwortliche Manager/-innen bzw. Eigentümer/-innen des 
Unternehmens, Mitarbeitende als vermeintliche Nutzende, Betriebs- und Personalräte, Einrichtungen und Verbände aus Forschung \& Entwicklung und Kund/-innen des Maschinenbaus (Hüsing, et al., 2002).

Im Projekt lag der Fokus auf der Einbindung von Mitarbeitenden aus verschiedenen Abteilungen. Andere Akteure z.B. Entscheider/-innen oder wissenschaftliche Mitarbeitende brachten ihre Perspektive als Teil des Projektteams ein. Im Sinne der Phase des Verstehens im UCD-Prozess wurde zum Start der Anforderungserhebung zunächst eine Vorortbesichtigung sowie ein umfangreiches Gespräch mit einem Vertreter der Akteursgruppe Prüffeldpersonal (Mitarbeitende) durchgeführt. Um einen Überblick zu schaffen, wurde im nächsten Schritt auf Basis der Besichtigung ein Online-Fragebogen entwickelt und an die verschiedenen Akteursgruppen versendet. Der Fragebogen beinhaltete zwei zentrale Fragestellungen:

- Welche Anforderungen stellen Akteur/-innen an die AR-Technologie und dessen Implementierungsprozess?

- Welche Aussagen können zur Technikakzeptanz getroffen werden?

Der Fokus dieser Arbeit liegt auf den Erkenntnissen zu Frage 1. Aufgrund pandemischer Rahmenbedingungen erwies sich dieser Einstieg als hilfreich, um einen ersten Eindruck zu gewinnen, der dann in Interviews vertieft wurde. Der erste Eindruck bietet eine Grundlage für weitere Erhebungen. Der Fragebogen wurde von 23 Teilnehmenden beantwortet und bestand aus 28 qualitativen und quantitativen Fragen aus den Rubriken: Maschinenabnahme, Technikakzeptanz und AR-Technologie.

Vergegenwärtigung: Bedingt durch die Corona-Pandemie haben Mitarbeitende des Anwenderunternehmens bereits eine provisorische Lösung zur Fernabnahme etabliert, die als eine Art Prototyp fungierte. An die Erfahrungen mit dem Prototypen konnte die Bedarfsabfrage anknüpfen. Die UCD-Phase der Vergegenwärtigung ersucht erste Ergebnisse z.B. über Visualisierungen/ Prototypen zu externalisieren. Prototypen, ob sprachlicher oder materieller Art, dienen als Repräsentationen von Wissen. Im Sinne psychologischer Lerntheorien vermag eine Idee, die bereits Teil eigener individueller Wissensstrukturen ist, als Ankerpunkt für Neues dienen, so dass Personen inhaltliche Beziehungen zwischen neuen Informationen und vorhandenem Wissen knüpfen können (Ausubel, 1974; Edelmann \& Wittmann, 2012; Seel, 2016). Daher erleichtern sichtbare Wissensrepräsentationen es den Akteur/-innen ihre Bedürfnisse zu verbalisieren und weitere hinzuzufügen. Dazu eignet sich im Anwendungsfall einerseits die provisorische Lösung der Mitarbeitenden. Andererseits entstand aus dem ersten Gespräch sowie den Ergebnissen des Fragebogens eine Visualisierung. Dazu 
wurden aus den überwiegend qualitativen Fragen des Fragebogens Anforderungen destilliert und kategorisiert. Anschließend wurden 21 User Stories ausformuliert. Aus den User Stories wurde das erste Mock-up als Design-Entwurf abgeleitet.

Evaluieren: Das Mockup wurde in sieben leitfadengestützten Einzelinterviews mit Akteur/-innen per Videoanruf in ca. einer Stunde diskutiert. Daraus entstanden angepasste, erweiterte und neue User Stories. Diese wiederum wurden in einem Workshop innerhalb des Projektkonsortiums diskutiert und priorisiert. Die Visualisierung als weitere Vergegenwärtigung dient in dieser Phase auch der teaminternen Kommunikation. Aus den User Stories entstand neben einem aktualisierten Mock-up ein Konzept, das Nutzerbedarfe mit den technischen Anforderungen verknüpft.

\section{Ergebnisse}

Aus dem in oben beschriebenen nutzerzentrierten Vorgehen wurden folgende Anforderungen an das System definiert:

1. Zugang zu Soll- und Ist-Messwerten sowie zu Toleranzwerten

2. Eine selbsterklärende Bedienoberfläche

3. Die Unterstützung eines verständlichen und interessanten Kundenerlebnisses

4. Uneingeschränkte Geschehensverfolgung

5. Der eigenständige Wechsel zwischen verschiedenen Streams (verschiedenen Kameraperspektiven, Datenvisualisierungen oder Überblendungen)

6. Verschiedene Detailansichten der Maschine

7. Möglichkeit zur Vorbereitung auf den Prüfablauf

8. Ablaufplan für die Abnahme

9. Ein Planungscenter zur Koordination des Prüfablaufes

Generell fiel es den Befragten schwer, Bedarfe an eine AR-Technologie zu formulieren. Zum einen gab die Mehrheit der Befragten an noch keine Erfahrungen mit ARTechnologien gemacht zu haben. Zum anderen wurden Herausforderungen durch die Befragten adressiert, die nicht über AR-Technologien zu lösen sind oder den Projektrahmen übertreffen würden bspw. eine bessere Datenverwaltung oder eine digitale Begehung der Maschine. Letzterem würde eher eine Virtual Reality Lösung entsprechen. Hilfreich war die Arbeit mit Beispielen und Visualisierungen in den Interviews. 


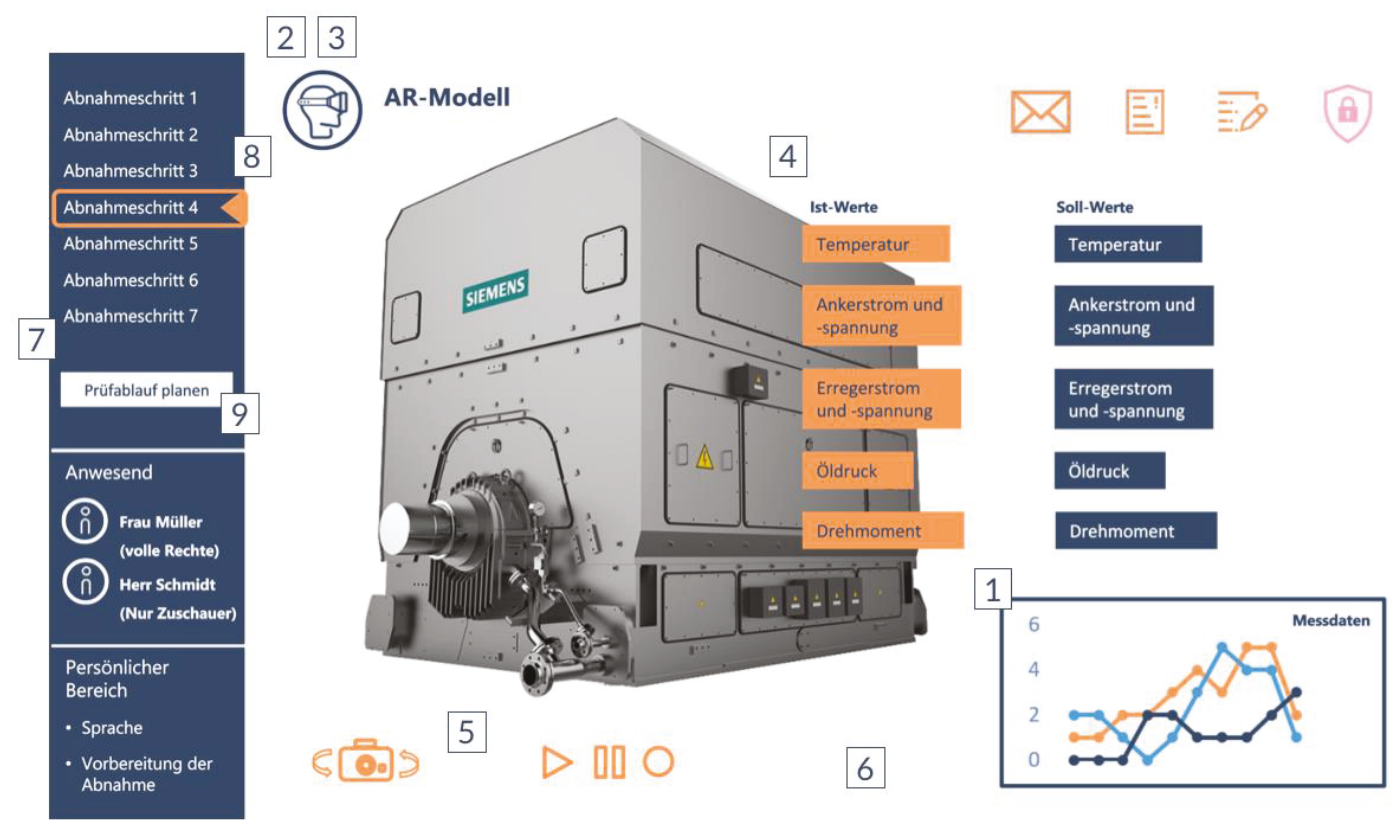

Abbildung 1: Finales Mock up der Bedienoberfläche, YOUSE GmbH (2021)

\section{Technisches Konzept einer AR-unterstützen Maschinenabnahme}

Parallel zur Erhebung der Nutzerbedarfe wurden technische Möglichkeiten ermittelt und ein Datenflusskonzept erstellt. Technische und nutzerspezifische Anforderungen flossen in ein Konzept zur technischen Umsetzung. Eine zentrale Anforderung ist, ein gleichwertiges Kundenerlebnis gegenüber der Abnahme vor Ort zu erzeugen, das eine detaillierte Geschehensverfolgung ermöglicht. AR eignet sich durch die Verknüpfung von realen Bildern und digitalen Informationen. So können Sichtprüfungen getätigt und durch überblendete Daten angereichert werden. Die Umsetzung einer AR-Technologie erfordert die Auswahl entsprechender Hardware. Aufgrund von Sicherheitsbedenken ist das Tragen von HMDs durch das Prüfpersonal während des Prüfprozesses ausgeschlossen. Stattdessen werden Mobilgeräte zur Augmentierung des Prüfprozesses verwendet. Dazu werden mehrere Mobilgeräte um den elektrischen Antrieb aufgestellt, damit die Maschine aus verschiedenen Perspektiven geprüft werden kann. Nach Möglichkeit wünschen sich Zuschauende selbstständig zwischen den verschiedenen Perspektiven und Detailansichten wechseln zu können. Damit Zuschauende digital an der Abnahme teilnehmen können, wird eine Verbindung zwischen den Mobiltelefonen und einem Server aufgebaut, welche die augmentierte Aufnahme auf eine webbasierte Bedienoberfläche streamt. Eine einfache Zugänglichkeit und Teilnahme 
am Abnahmeprozess ohne zusätzliche Hardware entsprechen den erhobenen Bedarfen. Spezielle Hardware ortsunabhängig zur Verfügung zu stellen, ist laut den Befragten keine Alternative. Um prüfungsnotwendige Daten über das Objekt zu überblenden, findet eine Objekterkennung anhand der 3D-Model-Target Methode des elektrischen Antriebs statt. Als prüfungsrelevante Daten zählen Ist-Werte der Sensorik und ausgewählte 3D-Modelle in Kombination mit Simulationsdaten. Die 3D-Modelle in Kombination mit Simulationsdaten dienen den Teilnehmenden dazu einen Vergleich zwischen Soll- und Ist-Dynamik nachvollziehen zu können.

Neben der Übertragung der Prüf-Szenerie ist der umfängliche Zugang zu Daten ein wichtiges Kriterium für Nutzende. Prüfungsrelevante Daten, wie Fertigungs-, Materialzeugnisse und Sollwerte, stehen für den Abgleich mit den Ist-Zuständen der webbasierten Bedienoberfläche zur Verfügung. Für die Datenanzeige müssen unterschiedliche Netzwerkakteure miteinander kommunizieren. Dazu werden die Sensordaten und Positionen sowie die 3D-Modelle und Simulationsdaten zwischen den Mobiltelefonen synchronisiert. Die Sensordaten des elektrischen Antriebs werden über einen Server an die AR-Geräte gesendet, damit diese überblendet werden. Soll-Werte, prüfungsrelevante Daten, 3D-Modelle und Simulationsmodelle sind auf dem Product Life Cycle Management (PLM)-Server hinterlegt. Mobiltelefone greifen Informationen von 3DModellen und Simulationsmodellen von einem PLM-Server ab. Die Soll-Werte und Prüfungsunterlagen werden vom PLM-Server auf den Prüffeldserver übertragen und anschließend auf einer webbasierten Bedienoberfläche angezeigt.

\section{Nachweis der AR-Funktion des Konzepts}

Um die grundlegenden AR-Funktionen des vorgestellten Konzepts nachzuweisen, wurde der elektrische Antrieb durch ein Pappmodell als Prototyp dargestellt. Für den Funktionsdemonstrator wurden Beispieldaten und -modelle erzeugt und auf zwei Mobiltelefonen gespeichert. Die Anbindung an den PLM-Server wurde vorerst ausgelassen. Die Mobiltelefone wurden an zwei Positionen befestigt. Das Objekt wurde mittels 3D-Model Target erkannt. Eingeblendet wurden eine Antriebswelle in Kombination mit Temperatursimulation. Die Temperatur ist abhängig von der Drehzahl. Zusätzlich werden Prüfschritte, -beschreibungen, Sollwerte und die Position der Sensorsollwerte über das Model geblendet. Drehzahl, Temperatur, Sensorpositionen und Prüfablaufschritte wurden zwischen den Mobiltelefonen synchronisiert. Eine Ansicht wird über ein Websocket an einen lokalen Server gesendet und über HTML dargestellt. Der erste Prototyp wurde pandemiebedingt wurde im Homeoffice erstellt und zeigt, dass die grundlegenden Funktionen der AR umsetzbar sind (Abb. 2 \& Abb. 3). 


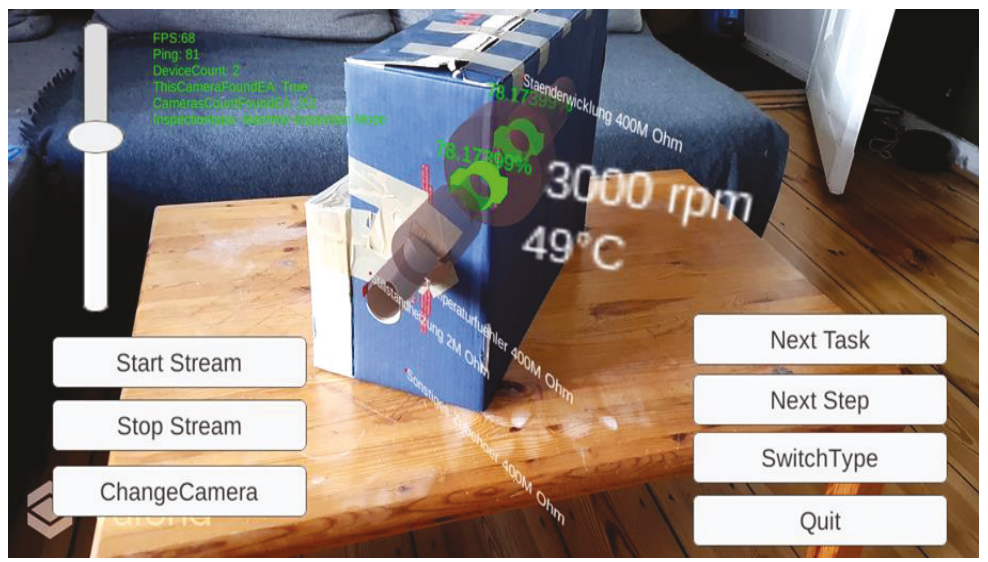

Abbildung 2: Nachweis der AR-funktionen für die Abnahme anhand eines Pappmodels, TU Berlin (2021)

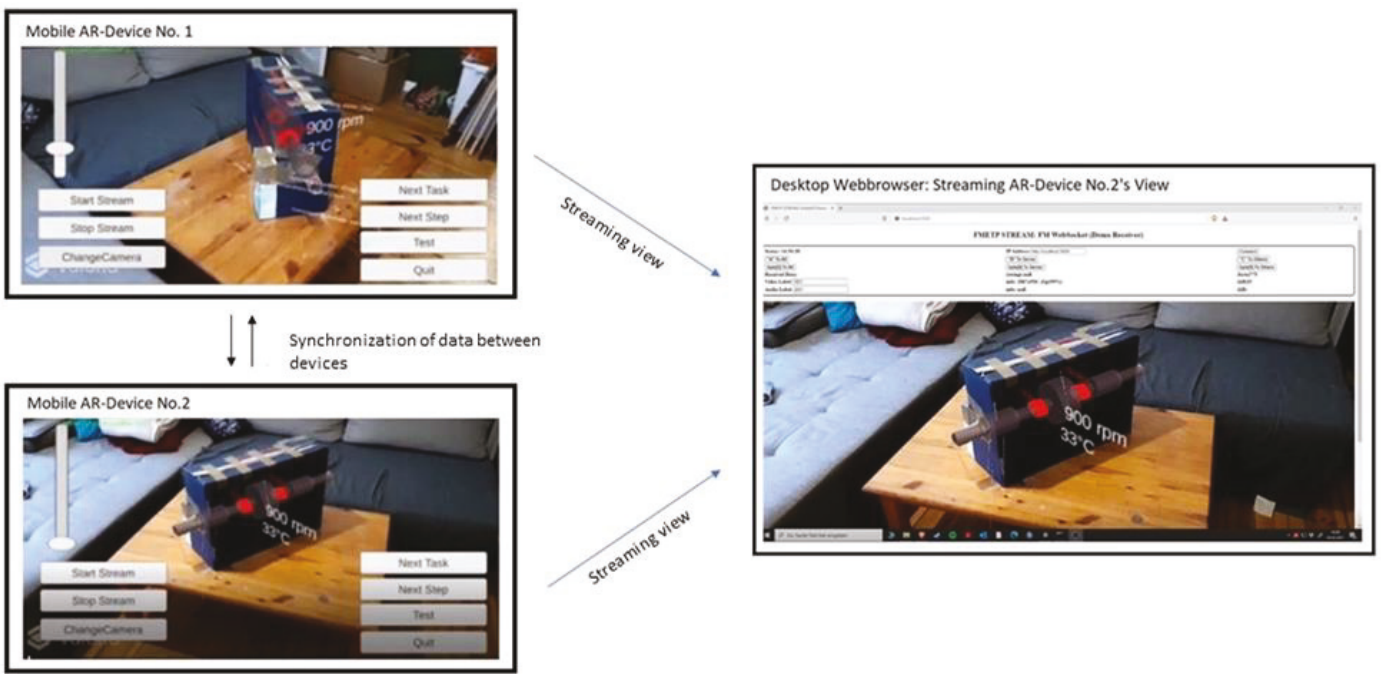

Abbildung 3: Zwei Mobiltelefone augmentieren dasselbe Objekt. Anschließend wird eine Ansicht an das HTML-Interface gesendet, TU Berlin (2021) 


\section{Fazit und Ausblick}

In dieser Arbeit wird ein Konzept für die AR-unterstützte, ortsunabhängige Abnahme von elektrischen Antrieben vorgestellt. Das Konzept ist in Zusammenarbeit zwischen Wissenschaftler/-innen aus Sozialwissenschaft und Ingenieurswissenschaft entstanden und beruht gleichermaßen auf technischen als auch Nutzeranforderungen. Aufgrund der besseren Visualisierung durch Augmented Reality können Prozesse durch Personal schneller und fehlerfreier durchgeführt werden als durch herkömmliche Verfahren, wie Arbeiten vor dieser bereits belegt haben. Aus technischer Sicht ist ein umsetzbares Konzept entstanden. Zudem ist es gelungen, dem Großteil der Nutzerbedarfe zu entsprechen, mit der Einschränkung, dass zu diesem Zeitpunkt nur wenige AR-spezifische Nutzeranforderungen erhoben werden konnten. Es ist daher ratsam, auch erste Prototypen von Nutzenden bewerten zu lassen. Im Anschluss an das Projekt ist zu überprüfen, ob der Demonstrator von Nutzenden akzeptiert wird, und ob der Prozess übersichtlicher, schneller und subjektiv angenehmer verläuft als mit herkömmlichen Prozessen. Das ist die Voraussetzung, um zu validieren, ob die ortsunabhängige Maschinenabnahme ein gleichwertiges oder verbessertes Kundenerlebnis bietet. Erschwerend erzwang die Pandemie, die Kommunikation weitestgehend digital auszuführen und Prototypen im privaten Umfeld statt im Labor zu erproben.

Diese Arbeit gilt als Grundstein für eine ortsunabhängige, virtuelle Inbetriebnahme. In Zukunft soll es hierbei möglich sein, die Maschine in einer rein virtuellen und digitalen Repräsentation vor der realen Inbetriebnahme zu testen.

\section{Literaturverzeichnis}

Ausubel, D. P. (1974): Psychologie des Unterrichts 1. Weinheim: Beltz.

Benyon, D. (2010): Designing interactive systems. Addison Wesley.

Brown, S. A., Massey, A. P., Montoya-Weiss, M. M. \& Burkman, J. R. (2002): Do I really have to? User acceptance of mandated technology. In: European Journal of Information Systems, 11(4), 283-295.

Bundesministerium für Bildung und Forschung (BMBF) (2018): Die neue Hightech Strategie: Technik zum Menschen bringen Forschungsprogramm zur Mensch-Technik-Interaktion. https://www.bmbf.de/upload filestore/pub/Technik zum Menschen bringen Forschungsprogramm.pdf, abgerufen am 28.02.2021.

Butz, A. \& Krüger, A. (2017): Mensch-Maschine-Interaktion. 2. Aufl. Berlin/Boston: Walter de Gruyter GmbH.

Deutsches Institut für Normung (2011): DIN EN ISO 9241-210:2011-01, Ergonomie der Mensch-System-Interaktion - Teil 210: Prozess zur Gestaltung gebrauchstauglicher interaktiver Systeme (ISO 9241-210:2010). Berlin: Deutsche Fassung EN ISO 9241210:2010. 
Edelmann, W. \& Wittmann, S. (2012): Lernpsychologie. 7. Aufl. Weinheim: Beltz.

Gemser, G. \& Perks, H. (2015): Co-Creation with Customers: An Evolving Innovation Research Field. In: Journal of Product Innovation Management, 5 (32), 660-665.

Hüsing, B., Bierhals, R., Bührlen, B., Friedewald, M., Kimpeler, S., Menrad, K., Wengel, J., Zimmer, R., Zoche, P. (2002):

Technikakzeptanz und Nachfragemuster als Standortvorteil. Karlsruhe: Fraunhofer-Institut für Systemtechnik und Innovationsforschung (ISI).

Kriegesmann, B. \& Kerka, F. (2014): Unternehmerisches Innovationsmanagement. In: M. Mai (Hrsg.): Handbuch Innovationen Interdisziplinäre Grundlagen und Anwendungsfelder. Wiesbaden: Springer Fachmedien, 73-87.

Lüthje, C. (2007): Methoden zur Sicherstellung von Kundenorientierung in den frühen Phasen des Innovationsprozesses. In: C. Herstatt \& B. Verworn (Hrsg.): Management der frühen Innovationsphasen. 2. Aufl. Wiesbaden: Gabler Verlag, 39-60.

Mehler-Bicher, A. \& Steige, L. (2014): Augmented Reality: Theorie und Praxis. 2. Aufl. München: Oldenbourg Wissenschaftsverlag.

Mura, M. D. \& Dini, G. (2015): Application of Augmented Reality Techniques in Through-life Engineering Services. In: Procedia CIRP, $38,14-23$.

Norman, D. A. \& Draper, S. W. (1986): User-Centered System Design: New Perspectives on Human-Computer Interaction. Hillsdale, NJ: Lawrence Earlbaum Associates.

Polvi, J., Taketomi , T. \& Moteki, A. (2018): Handheld Guides in Inspection Tasks. In: IEEE Transactions on Visualization and Computer Graphics, 24 (7), 2118-2128

Renn, 0. (2005): Technikakzeptanz: Lehren und Rückschlüsse der Akzeptanzforschung für die Bewältigung des technischen Wandels. In: TATuP - Zeitschrift für Technikfolgenabschätzung in Theorie und Praxis, 14 (3), 29-38.

Revetria, R., Tonelli, F., Damiani, L. \& Demartini, M. (2019): A Real-time Mechanical Structures Monitoring System Based on Digital Twin, IoT and Augmented Reality. San Diego: ANSS' 19 Proceeding of the Annual Simulation Symposium, 18, 1-10

Schroeder, G., Steinmetz, C., Garcia, N. \& Espindola, D. (2016): Visualising the Digital Twin using Web Services as Web Service, Poiteres, France: IEEE 14th International Conference on Industrial Informatics.

Seel, N. M. (2016): Psychologie des Lernens. Lehrbuch für Pädagogen und Psychologen. 2. Aufl. Stuttgart: UTB GmbH.

Stark, E. , Kucera, E., Haffner, O., Draho, P., Leskovsky, R. (2020): Using Augmented Reality and Internet of Things for Control and Monitoring of Mechatronic Devices, Electronics 2020, 9(8), 1272

Trübswetter, A., Figueiredo, L. \& Prinz, F. (2020): Gestaltung Integrierter Forschung Ansätze zur ganzheitlichen Nutzerintegration. In: Gransche, B.; Manzeschke, A. (Hrsg.): Das geteilte Ganze Horizonte Integrierter Forschung für künftige Mensch-Technik-Verhältnisse. Wiesbaden: Springer Fachmedien, 305-324.

Vredenburg, K., Mao, J.-Y., Smith, P. W. \& Carey, T. (2002): Practice, A Survey of User-Centered Design. In: Proceedings of the SIGCHI Conference on Human Factors in Computing Systems, 471-478.

Webster, A., Feiner, S., Macintyre, B., Massie, W., Krueger, T. (1996): Augmented Reality in Architectural Construction, Inspection, and Renovation. In: Proc. ASCE Third Congress on Computing in Civil Engineering, 996.

Williams, M., Yao, K. K. K. \& Nurse, J. R. C. (2020): Cornell University: Developing an Augmented Reality Tourism App through UserCentred Design (Extended Version). https://arxiv.org/abs/2001.11131, abgerufen am 13.03.2021. 
Werner von Siemens Centre (WvSC) (2020): Elektrische Antriebe Forschungsprojekt Produktionstechnischer Wandel. https://wvsc.berlin/forschungsprojekt-elektrische-antriebe/, abgerufen am 28.02.2021.

Zheng, L., Liu, X., An, Z., Li, Z., Zhang, R. (2019): A smart assistance system for cable assembly by combining wearable augmented reality with portable visual inspection. In: Virtual Reality \& Intelligent Hardware 2 (1), $12-27$.

Zhou, J., Lee, I., Thomas, B. \& Mena, R. (2012): Applying Spatial Augmented Reality to Facilate. In: Situ Support for Automotive Spot Welding, New York, NY, The International Journal of Virtual Reality, 11(1):33-41

\section{Projektinformationen}

Das Projekt EA 2.0 ist kofinanziert durch den Europäischen Fonds für regionale Entwicklung (EFRE) und findet im Rahmen des Werner von Siemens Centre - for Industry and Scienece (WvSC).

\section{Kontakt}

Nedim Kovacevic, M. Sc.

Prof. Dr.-Ing. Rainer Stark

Technische Universität Berlin

Institut für Werkzeugmaschinen und Fabrikbetrieb

Pascalstraße 8-9

10587 Berlin

https://www.iit.tu-berlin.de/menue/industrielle_informationstechnik/

Jantje Meinzer, M. Sc.

YOUSE GmbH

Florastraße 47

13187 Berlin

https://youse.de/ 